\title{
Tree domination polynomial of some graphs
}

\author{
Rafia Yoosuf ${ }^{1 *}$ and Preethi Kuttipulackal ${ }^{2}$
}

\begin{abstract}
The tree domination polynomial of a connected graph $G$ of order $n$ is the polynomial $D_{t d}(G, x)=$ $n \sum i=\gamma_{t d}(G) d_{t d}(G, i) x^{i}$, where $d_{t d}(G, i)$ is the number of tree dominating sets of $G$ of cardinality $i$ and $\gamma_{t d}(G)$ is the tree domination number of $G$. In this paper the $t d$ - polynomial of some graphs are studied.

Keywords

Domination, Tree domination, domination polynomial, Tree domination polynomial $(t d-$ polynomial).

AMS Subject Classification 05 C69.

${ }^{1}$ Department Of Mathematics, M E S Mampad College, Kerala, India.

2 Department Of Mathematics, University Of Calicut, Kerala, India.

*Corresponding author: ${ }^{1}$ rafiafiroz@gmail.com; ${ }^{2}$ pretikut@rediffmail.com

Article History: Received 24 January 2019; Accepted 24 May 2019

(C)2019 MJM
\end{abstract}

\section{Contents}

1 Introduction 451

2 Tree Domination Polynomial... 451

3 References 456 456

\section{Introduction}

The domination polynomial of a graph is introduced by Saeid Alikhani and Yee-hock Peng in [5]. While extending the concept of domination polynomial in view of tree dominating set, we came across with many interesting relations with the coefficients of the polynomial and the graph parameters. Also, the coefficients of the polynomial of some important class of graphs have attractive patterns and the roots of the polynomial have interesting nature. This paper includes the $t d-$ polynomial of some class of graphs.

Let $G=(V, E)$ be a simple graph. For any vertex $v \in V$, the open neighbourhood of $v$ is the set $N(v)=\{u \in V$ : $u v \in E\}$ and the closed neighbourhood of $v$ is the set $N[v]=$ $N(v) \bigcup\{v\}$. For a set $S \subseteq V$, the open neighbourhood of $S$ is $N(S)=\cup_{v \in S} N(v)$ and the closed neighbourhood of $S$ is $N[S]=N(S) \cup S$. A set $S \subseteq V$ is a dominating set of $G$, if $N[S]=V$, or equivalently every vertex $\operatorname{in} V \backslash S$ is adjacent to atleast one vertex in $S$. The domination number $\gamma(G)$ is the minimum cardinality of a dominating set in $G$. A dominat- ing set with cardinality $\gamma(G)$ is called a $\gamma-$ set. Let $G$ be a simple connected graph of order $n$. A tree dominating set of $G$ is a set $S$ of vertices of $G$ such that every vertex in $V \backslash S$ is adjacent to some vertex in $S$ and the induced subgraph $\langle S>$ is tree. The tree domination number $\gamma_{t d}(G)$ is the minimum cardinality of a tree dominating set in $G$.

Saeid Alikhani and Yee-hock Peng introduced the concept of domination polynomial of a graph as the polynomial $D(G, x)=\sum_{i=\gamma(G)}^{n} d(G, i) x^{i}$, where $d(G, i)$ denotes the number of dominating sets of cardinality $i$.

Considering the polynomial idea of Alikhani et.al., we introduced the $t d$ - polynomial of a connected graph and study the information about the graph that we can obtain from the polynomial. For the basic concepts in graph theory we refer mainly Bondy and Murthy[1]. The graphs considered here are all connected and simple of order $n$.

\section{Tree Domination Polynomial}

As the elements of a $t d-$ set form a tree, the maximum cardinality of $t d-$ set may be $n$, and the minimum cardinality is $\gamma_{t d}(G)$.

Definition 2.1. Let $G$ be a connected graph of order $n$. The tree domination polynomial of $G$ is the polynomial $D_{t d}(G, x)=$ 


$$
\begin{aligned}
& i=\gamma_{t d}(G) \\
& \text { dominating sets of } G \text { of cardinality } i .
\end{aligned}
$$

Example 2.2. Consider the path $P_{3}=v_{1} v_{2} v_{3}$.

Then the tree domination number is one and the tree dominating set of size one is $\left\{v_{2}\right\}$, the tree dominating set of size two are $\left\{v_{1}, v_{2}\right\},\left\{v_{2}, v_{3}\right\}$, the tree dominating sets of size three is $\left\{v_{1}, v_{2}, v_{3}\right\}$, So that the polynomial is

$$
x+2 x^{2}+x^{3} .
$$

A dominating set $S$ is called a tree dominating set if the induced subgraph $\langle S\rangle$ is a tree.

\section{Observations}

Let $G$ be a connected graph with $n$ vertices then

1. If $G$ contains a cycle then $d_{t d}(G, n)=0$. Because if we take all the vertices then the induced graph won't form a tree.

2. For any tree $d_{t d}(G, n)=$ The number of pendant(end) vertices(end).

3. For any tree $d_{t d}(G, n-1)=n-1$.

4. $D_{t d}(G, x)$ has no constant term.

5. Zero is a root of $D_{t d}(G, x)$ with multiplicity $\gamma_{t d}(G)$.

Theorem 2.3. For any path $P_{n}, n \geq 3$ vertices, $D_{t d}\left(P_{n}, x\right)=x^{n-2}+2 x^{n-1}+x^{n}$.

Proof. Let $P_{n}$ be the path and $\left\{v_{1}, v_{2}, \ldots, v_{n}\right\}, n \geq 3$. The tree domination number is $n-2$ and there is only one tree dominating set of order $n-2$. That means $d_{t d}\left(P_{n}, n-2\right)=$ 1. Also there are only two tree dominating sets of order $n-1$ namely $\left\{v_{1}, v_{2}, \ldots \ldots v_{n-1}\right\}$ and $\left\{v_{2}, v_{3}, \ldots v_{n}\right\}$. Therefore $d_{t d}\left(P_{n}, n-1\right)=2$ and there is only one tree dominating sets of order $n$.

This completes the proof.

Theorem 2.4. For any cycle $C_{n}, n \geq 3$ vertices, $D_{t d}\left(C_{n}, x\right)=n x^{n-1}+n x^{n-2}$.

Proof. Let $C_{n}=\left\{v_{1}, v_{2}, \ldots, v_{n}, v_{1}\right\}, n \geq 3$. The tree domination number is $n-2$. Removal of any one vertex from $V$ will yeild a tree dominating set of cardinality $n-1$ and removal of two adjacent vertices will give a tree dominating set of cardinality $n-2$ so that $d_{t d}\left(C_{n}, n-1\right)=n$ and $d_{t d}\left(C_{n}, n-2\right)=n$.

This completes the proof.

Theorem 2.5. For any complete graph $K_{n}, n \geq 3$, $D_{t d}\left(K_{n}, x\right)=\left(\begin{array}{l}n \\ 1\end{array}\right) x+\left(\begin{array}{l}n \\ 2\end{array}\right) x^{2}$.

Proof. Let $G$ be a complete graph with $n$ vertices $\left\{v_{1}, v_{2}, \ldots, v_{n}\right\}$ and $\gamma_{t d}\left(K_{n}\right)=1$. There are $n$ tree dominating sets of order 1. Any set of three or more vertices induce a cycle i.e. $\gamma_{t d}\left(K_{n}, m\right)=0, m \geq 3$. Also as any two vertices induce a tree there are $\left(\begin{array}{l}n \\ 2\end{array}\right)$ tree domination set of cardinality 2 . This completes the proof.
Theorem 2.6. For any star graph $K_{1, t}, t \geq 2, D_{t d}\left(K_{1, t}, x\right)=$ $x+\left(\begin{array}{l}t \\ 1\end{array}\right) x^{2}+\left(\begin{array}{l}t \\ 2\end{array}\right) x^{3}+\left(\begin{array}{l}t \\ 3\end{array}\right) x^{4}+\ldots+\left(\begin{array}{c}t \\ t-1\end{array}\right) x^{t}+\left(\begin{array}{l}t \\ t\end{array}\right) x^{t+1}=$ $x(1+x)^{t}$.

Proof. Let $K_{1, t}$ be a star graph with vertices $\left\{v, v_{1}, v_{2}, \ldots, v_{t}\right\}$, where $v$ is the centre vertex of the graph. Since $t \geq 2$ any tree dominating set must contain the centre vertex $v$, so that there is only one tree dominating set of order 1 namely $\{v\}$, there are $\left(\begin{array}{l}t \\ 1\end{array}\right)$ tree dominating sets of cardinality 2 , and so on. Therefore $D_{t d}\left(K_{1, t}, x\right)=x+\left(\begin{array}{l}t \\ 1\end{array}\right) x^{2}+\left(\begin{array}{l}t \\ 2\end{array}\right) x^{3}+\left(\begin{array}{l}t \\ 3\end{array}\right) x^{4}+$ $\ldots+\left(\begin{array}{c}t \\ t-1\end{array}\right) x^{t}+\left(\begin{array}{l}t \\ t\end{array}\right) x^{t+1}=x(1+x)^{t}$. This completes the proof.

Theorem 2.7. The tree domination polynomial of $K_{m, n}$ for $1<m \leq n$ is

$D_{t d}\left(K_{m, n}, x\right)=\left(\begin{array}{c}m \\ 1\end{array}\right)\left(\begin{array}{l}n \\ 1\end{array}\right) x^{2}+\sum_{k=3}^{m+1}\left[\left(\begin{array}{c}m \\ 1\end{array}\right)\left(\begin{array}{c}n \\ k-1\end{array}\right)+\left(\begin{array}{c}n \\ 1\end{array}\right)\left(\begin{array}{c}m \\ k-1\end{array}\right)\right] x^{k}$

$+\sum_{k=m+2}^{n+1}\left(\begin{array}{c}m \\ 1\end{array}\right)\left(\begin{array}{c}n \\ k-1\end{array}\right) x^{k}$.

Proof. $\gamma_{t d}\left(K_{m, n}\right)=2$. There are $\left(\begin{array}{l}m \\ 1\end{array}\right)\left(\begin{array}{l}n \\ 1\end{array}\right)$ tree dominating sets of cardinality 2 , because we cannot choose both these vertices from the same bipartite set of vertices as it wont dominate the remaining vertices of the same bipartite set. There are $\left[\left(\begin{array}{c}m \\ 1\end{array}\right)\left(\begin{array}{l}n \\ 2\end{array}\right)+\left(\begin{array}{c}m \\ 2\end{array}\right)\left(\begin{array}{c}n \\ 1\end{array}\right)\right]$ choices for tree dominating sets of cardinality 3 . If we choose two or more vertices from both bipartite sets then the induced subgraph of these vertices will form a cycle. So that a tree dominating set cannot contain two vertices of each bipartite set. Therefore at a time we can choose one vertex from either of the two bipartite set and the remaining ones from the other set of vertices $(*)$. So there are $\left[\left(\begin{array}{c}m \\ 1\end{array}\right)\left(\begin{array}{c}n \\ k-1\end{array}\right)+\left(\begin{array}{c}m \\ k-1\end{array}\right)\left(\begin{array}{c}n \\ 1\end{array}\right)\right]$ choices of tree dominating sets of order $k$ elements, for $k \leq m+1$. Now for $k \geq m+2$, we have to choose $k-1 \geq m+1$ vertices from the bipartite set of $n$ elements and one vertex from the other set(because of $*$ ). That gives $\left(\begin{array}{c}n \\ k-1\end{array}\right)\left(\begin{array}{c}m \\ 1\end{array}\right)$ tree dominating sets. Again by $*$, the number of vertices in a tree dominating set cannot exceed $n+1$. Note that for $1<m \leq n$, the polynomial of $K_{m, n}$ can be expressed as $D_{t d}\left(K_{m, n}, x\right)=\left(\begin{array}{c}m \\ 1\end{array}\right)\left(\begin{array}{c}n \\ 1\end{array}\right) x^{2}+\sum_{k=3}^{n+1}\left[\begin{array}{c}m \\ 1\end{array}\right)\left(\begin{array}{c}n \\ k-1\end{array}\right)+$ $\left.\left(\begin{array}{c}n \\ 1\end{array}\right)\left(\begin{array}{c}m \\ k-1\end{array}\right)\right] x^{k}, \rightarrow(1)$ where $\left(\begin{array}{c}m \\ k-1\end{array}\right)=0$ for $k-1>m$, that is for $k>m+1$. Also note that when $m=1, K_{m, n}$ is a star graph. But the expression is not true. However if $m=1$, and $n>1$ $D_{t d}\left(K_{m, n}, x\right)=\sum_{k=1}^{n+1}\left(\begin{array}{c}m \\ 1\end{array}\right)\left(\begin{array}{c}n \\ k-1\end{array}\right) x^{k}$ with an additional term $x$ in equation (1).

This completes the proof.

A complete m-partite graph $K_{n_{1}, n_{2}, \ldots, n_{m}}$ is a graph whose vertex set can be partitioned into m non-empty sets $V_{i}, i=$ $1,2, \ldots, m$ with $\left|V_{i}\right|=n_{i}$ such that every vertex in $V_{i}$ is adjacent to every vertex in $V_{j}$ for every $i \neq j$ and $i, j \in\{1,2, \ldots, m\}$. 
Theorem 2.8. For a complete m-partite graph $K_{n_{1}, n_{2}, \ldots, n_{m}}$, with $1<n_{1} \leq n_{2} \leq \ldots \leq n_{m} ; D_{t d}\left(K_{n_{1}, n_{2}, \ldots, n_{m}}, x\right)=\sum_{k=2}^{n_{m}+1} a_{k} x^{k}$, where $a_{2}=n_{1} \sum_{j>1} n_{j}+n_{2} \sum_{j>2} n_{j}+\ldots+n_{m-1} n_{m}=$ $\sum_{i=1}^{m-1}\left(n_{i} \sum_{j>i} n_{j}\right)$ and $\left.a_{k}=\sum_{i=1}^{m}\left[\left(\begin{array}{c}n_{i} \\ C_{i}\end{array}\right)\right)\left(\sum_{j \neq i}\left(\left(\begin{array}{c}n_{j} \\ C_{k-1}\end{array}\right)\right)\right)\right] \rightarrow(1)$, for $k>2$; with the convention that $\left(\begin{array}{c}n_{j} \\ C_{k}\end{array}\right)=0$ for $k>n_{j}$. If $n_{i}=1$ for $k$ vertices of $i$, then $D_{t d}(G, x)=k x+\sum_{k=2}^{n_{m}+1} a_{k} x^{k}$, where $a_{k}, k>2$ gives as in (1).

Proof. Since $m>1$, any two vertices from two different partite sets form a tree domination set and $\gamma_{t d}\left(K_{n_{1}, n_{2}, \ldots, n_{m}}\right)=2$. Also at a time we cant choose three or more vertices from three or more different partite sets because the induced subgraph of these vertices will form a cycle so it wont form a $t d-$ set. So that for a td-set of cardinality 2, we have $\left[\left(\begin{array}{l}n_{1} \\ c_{1}\end{array}\right)\left(\begin{array}{c}n_{2}+n_{3}+\ldots+n_{m} \\ c_{1}\end{array}\right)+\right.$ $\left.\left(\begin{array}{c}n_{2} \\ c_{1}\end{array}\right)\left(\begin{array}{c}n_{3}+\ldots+n_{m} \\ c_{1}\end{array}\right)+\ldots+\left(\begin{array}{c}n_{m-1} \\ c_{1}\end{array}\right)\left(\begin{array}{c}n_{m} \\ c_{1}\end{array}\right)\right]$ choices. For $t d-$ sets of cardinality 3 , we can choose one vertex from any of the partite sets namely $V_{j}$ and the other two vertices both from one partite set other than $V_{j}$. Therefore there are $\left(\begin{array}{l}n_{1} \\ c_{1}\end{array}\right)\left[\left(\begin{array}{l}n_{2} \\ c_{2}\end{array}\right)+\left(\begin{array}{l}n_{3} \\ c_{2}\end{array}\right)+\right.$ $\left.\ldots+\left(\begin{array}{c}n_{m} \\ c_{2}\end{array}\right)\right]+\left(\begin{array}{c}n_{2} \\ c_{1}\end{array}\right)\left[\left(\begin{array}{l}n_{1} \\ c_{2}\end{array}\right)+\left(\begin{array}{c}n_{3} \\ c_{2}\end{array}\right)+\ldots+\left(\begin{array}{c}n_{m} \\ c_{2}\end{array}\right)\right]+\ldots+\left(\begin{array}{c}n_{m} \\ c_{1}\end{array}\right)\left[\left(\begin{array}{l}n_{1} \\ c_{2}\end{array}\right)+\right.$ $\left.\left(\begin{array}{l}n_{2} \\ c_{2}\end{array}\right)+\ldots+\left(\begin{array}{c}n_{m-1} \\ c_{2}\end{array}\right)\right]$ td-sets of cardinality 3 . Similarly there are $\left(\begin{array}{l}n_{1} \\ c_{1}\end{array}\right)\left[\left(\begin{array}{l}n_{2} \\ c_{3}\end{array}\right)+\left(\begin{array}{l}n_{3} \\ c_{3}\end{array}\right)+\ldots+\left(\begin{array}{l}n_{m} \\ c_{3}\end{array}\right)\right]+\left(\begin{array}{l}n_{2} \\ c_{1}\end{array}\right)\left[\left(\begin{array}{l}n_{1} \\ c_{3}\end{array}\right)+\left(\begin{array}{l}n_{3} \\ c_{3}\end{array}\right)+\ldots+\left(\begin{array}{l}n_{m} \\ c_{3}\end{array}\right)\right]+$ $\ldots+\left(\begin{array}{l}n_{m} \\ c_{1}\end{array}\right)\left[\left(\begin{array}{l}n_{1} \\ c_{3}\end{array}\right)+\left(\begin{array}{l}n_{2} \\ c_{3}\end{array}\right)+\left(\begin{array}{l}n_{3} \\ c_{3}\end{array}\right)+\ldots+\left(\begin{array}{c}n_{m-1} \\ c_{3}\end{array}\right)\right] t d$-sets of cardinality 4 .

if $k>n_{j}+1$ for $j=1,2, \ldots, p$ we have to choose one vertex from a partite set and the remaining $k-1$ vertices all from another single partite set. As $k-1$ vertices cannot be choosen together from partite sets $V_{1}, \ldots, V_{p}$; the coefficient of $x^{k}$ is

$\left(\begin{array}{l}n_{1} \\ c_{1}\end{array}\right)\left[\sum_{j>p}\left(\begin{array}{c}n_{j} \\ c_{k-1}\end{array}\right)\right]+\ldots+\left(\begin{array}{c}n_{p} \\ c_{1}\end{array}\right)\left[\sum_{j>p}\left(\begin{array}{c}n_{j} \\ c_{k-1}\end{array}\right)\right]$

$+\left(\begin{array}{c}n_{p+1} \\ c_{1}\end{array}\right)\left[\sum_{j>p+1}\left(\begin{array}{c}n_{j} \\ c_{k-1}\end{array}\right)\right]+\left(\begin{array}{c}n_{p+2} \\ c_{1}\end{array}\right)\left[\sum_{j>p, j \neq p+2}\left(\begin{array}{c}n_{j} \\ c_{k-1}\end{array}\right)\right]+\ldots+$

$\left(\begin{array}{c}n_{m} \\ C_{1}\end{array}\right)\left[\sum_{p<j<m}\left(\begin{array}{c}n_{j} \\ c_{k-1}\end{array}\right)\right]=\sum_{n_{1}}^{n_{m}}\left[\left(\begin{array}{c}n_{i} \\ c_{1}\end{array}\right)\left(\sum_{j \neq i}\left(\begin{array}{c}n_{j} \\ c_{k-1}\end{array}\right)\right]\right.$, where $\left(\begin{array}{c}n_{j} \\ c_{k-1}\end{array}\right)=$ 0 for $n_{j}<k-1$. Thus we can conclude that $a_{k}=$ $\sum_{i}\left(\left(\begin{array}{l}n_{i} \\ c_{1}\end{array}\right)\left[\sum_{j \neq i}\left(\begin{array}{c}n_{j} \\ c_{k-1}\end{array}\right)\right]\right)$, for $k \geq 3$. Since $n_{1} \leq n_{2} \leq \ldots \leq n_{m}$, the maximum cardinality of a td-set is $n_{m}+1$; which completes the arguments.

Theorem 2.9. For a complete $m$ partite graph $K_{n, n, \ldots, n}$, where $n$ repeats $m$ times,

$D_{t d}\left(K_{n, n, \ldots, n}, x\right)=m\left\{\left[\left(n C_{1}\right)\left((m-1) n C_{1}\right] x^{2}+\left[\left(n C_{1}\right)\left(n C_{2}\right)\right] x^{3}+\right.\right.$ $\left.\left[\left(n C_{1}\right)\left(n C_{3}\right)\right] x^{4}+\ldots+\left[\left(n C_{1}\right)\left(n C_{n}\right)\right] x^{n+1}\right\}$

A friendship graph $F_{n}$ is the one point union of $\mathrm{n}$ copies of the cycle $C_{3}$. Equivalently $F_{n}=n P_{2} \circ K_{1}$. When $\mathrm{n}=1$, $F_{n}=K_{3}=C_{3}$.

Theorem 2.10. For $n>1, D_{t d}\left(F_{n}, x\right)=\sum_{k=1}^{n+1} a_{k} x^{k}$, where $a_{k}=$ $2^{k-1}\left(\begin{array}{c}n \\ k-1\end{array}\right)$.
Proof. $\gamma_{t d}\left(F_{n}\right)=1$.

Let $\mathrm{v}$ be the apex vertex of $F_{n}$ common to all $C_{n}$. Since $n>1$, every td-set must contain the vertex $\mathrm{v}$ and no $t d$-set can contain all the three vertices from same block of $F_{n}$. There is only one $t d-$ set of one element, $\{v\}$; so that $a_{1}=1$. Now, $v$ together with any other vertex will form a $t d-$ set of 2 elements, and hence $a_{n}=2 n$.

In general, a td-set of $k$ elements is obtained by taking $v$ along with $k-1$ vertices from $k-1$ blocks of $F_{n}$. The $k-1$ blocks can be selected in $\left(\begin{array}{c}n \\ k-1\end{array}\right)$ ways, and for selecting one vertex from each block we have $2^{k-1}$ choices. So that $a_{k}=$ $2^{k-1}\left(\begin{array}{c}n \\ k-1\end{array}\right)$; which completes the proof.

A tadpole $T_{(n, l)}, n \geq 3, l \geq 1$ is a graph obtained by joining one of the end vertices of the path $p_{l}$ to any one of the vertices of the cycle $C_{n}$ by a bridge. The graph $T_{(n, l)}$ has $n+l$ vertices and $n+l$ edges.

Theorem 2.11. $D_{t d}\left(T_{(n, l)}, x\right)=(n-2) x^{n+l-3}+(2 n-3) x^{n+l-2}+$ $(n-1) x^{n+l-1}$

Proof. Let $v_{1}$ be the vertex of the cycle $C_{n}=v_{1} v_{2} \ldots v_{n} v_{1}$ to which the path $P_{l}=u_{1} u_{2} \ldots u_{l}$ is attached. Since $v_{1}$ is the only vertex connecting $C_{n}$ and $P_{l}, v_{1}$ should be a member of every $t d-$ set of a tadpole graph. Atleast $n-2$ vertices are required from $\left\{v_{1}, \ldots, v_{n}\right\}$ to form a tree dominating set and all the $n$ vertices cannot be included. So that from $\left\{v_{1}, \ldots, v_{n}\right\}$, we have to choose either $n-2$ vertices including $v_{1}$ or $n-1$ vertices including $v_{1}$. There are $n-2$ choices for choosing $n-2$ vertices and $n-1$ choices for $n-1$ vertices.

Similarly if the vertex in $P_{l}$ adjacent to $v_{1}$ is $u_{1}$, then a $t d-$ set of $T_{(n, l)}$ must include either all the vertices $u_{1}, \ldots, u_{l}$ or the 1-1 vertices $u_{1}, \ldots, u_{l-1}$.

Thus $T_{(n, l)}$ has $t d-$ sets of cardinalities

1. $n+l-3\left(\right.$ when $n-l v_{i}^{\prime} s$ and 1-1 $\left.u_{j}^{\prime} s\right)$,

2. $n+l-2$ (when $n-2 v_{i}^{\prime} s$ and $1 u_{j}^{\prime} s$ or n- $1 v_{i}^{\prime} s$ and $1-1 u_{j}^{\prime} s$ and

3. $n+l-1\left(\right.$ when $n-1 v_{i}^{\prime} s$ and $\left.1 u_{j}^{\prime} s\right)$

There are $n-2$ choices in category (1), $n-2+n-1=2 n-3$ choices in category (2) and $n-1$ choices in category (3). So the polynomial is $(n-2) x^{n+l-3}+(2 n-3) x^{n+l-2}+(n-$ 1) $x^{n+l-1}$ This completes the proof.

The n-barbell graph $B_{n, 1}$ is a graph obtained by connecting two copies of complete graph $K_{n}$ by a bridge. $B_{1,1}=K_{2}=P_{2}$ and $B_{2,1}=P_{4}$.

Theorem 2.12. For $n \geq 2 D_{t d}\left(B_{n, 1}, x\right)=x^{2}+2(n-1) x^{3}+$ $(n-1)^{2} x^{4}$.

Proof. Since $n \geq 2, \gamma_{t d}\left(B_{n, 1}\right)=2$ Let $u v$ be the bridge connecting two $K_{n}^{\prime} s$. Any $t d$-set of a n-barbell graph contains the two vertices $u$ and $v$. The only minimal $t d$-set is $\{u, v\}$; so that the coefficient of $x^{2}$ in $D_{t d}\left(B_{n, 1}, x\right)=1$. There are $t d-$ sets of 3 elements consisting of $u, v$ and any other vertex; so 
that the coefficient of $x^{3}$ is $2(n-1)$. The td-sets of cardinality 4 consisting of $u, v$ and two other vertices from two different blocks. So the coefficient of $x^{4}$ is $(n-1)^{2}$. As more than two vertices from a block constitute a cycle, these are the only $t d-$ sets.

In the n-barbel graph $B_{n, 1}$, two $K_{n}^{\prime} s$ are joined by an edge. If we consider the graph $K_{n}$ and $K_{m}$ by an edge, the arguments similar to the above, show the following.

Theorem 2.13. If $G$ is the graph obtained by connecting two complete graphs $K_{m}$ and $K_{n}$ by an edge, where $m, n>1$, then $D_{t d}(G, x)=x^{2}+(m+n-2) x^{3}+(m-1)(n-1) x^{4}$.

A bistar graph $B_{m, n}$ is obtained by connecting the center vertices of two star graphs $K_{1, m}$ and $K_{1, n}$ by an edge.

Theorem 2.14. For $m, n \geq 1, D_{t d}\left(B_{m, n}, x\right)=\sum_{k=2}^{m+n+2} a_{k} x^{k}$, where $a_{k}=\left(\begin{array}{c}m+n \\ k-2\end{array}\right)$.

Proof. Let the center vertex of the two star graphs $K_{1, m}$ and $K_{1, n}$ be $u$ and $v$ respectively. Also any $t d-$ set of $B_{m, n}$ must contain the two vertices $u$ and $v$ otherwise the induced subgraph wont form a tree and $\gamma_{t d}\left(B_{m, n}\right)=2$. There is only one $t d$-set of order 2 namely $\{u, v\}$. For td-sets of $k>2$ elements, we can consider any $k$-subset of the vertex set containing $u$ and $v$. As there are $m+n$ vertices other than $u$ and $v$, we have $\left(\begin{array}{c}m+n \\ k-2\end{array}\right) t d$-sets of $k$ elements; hence proved. This completes the proof.

A bistar graph $B_{n, n}$ is the union of two star graphs $K_{1, n}$ with centers $u$ and $v$ together with a new edge $u v$.

In the bistar graph $B_{m, n}$, two star graphs $K_{1, n}$ and $K_{1, m}$ are joined by an edge. If we consider the graph obtained by connecting two star graphs $K_{1, n}$ by an edge, the arguments similar to the above show the following.

Theorem 2.15. For a bistar graph $D_{t d}\left(B_{n, n}, x\right)=\sum_{k=2}^{2 n+2} a_{k} x^{k}$, where $a_{k}=\left(\begin{array}{c}2 n \\ k-2\end{array}\right)$.

A lollipop graph $L_{n, 1}$ is obtained by joining $K_{n}$ to a path of length 1 with a bridge.

Theorem 2.16. For a lollipop graph $L_{n, 1} D_{t d}\left(L_{n, 1}, x\right)=x+$ $n x^{2}+(n-1) x^{3}$.

Proof. Let the vertices of $L_{n, 1}$ be $v, v_{1}, \ldots, v_{n}$, where $\left\{v_{1}, \ldots, v_{n}\right\}$ induces $K_{n}$ and $v$ is adjacent to $v_{1}$. Any td-set of $L_{n, 1}$ must include the vertex $v_{1}$. The set $\left\{v_{1}\right\}$ is the only singleton $t d$-set. We can't choose more than two vertices from $K_{n}$ because the induced subgraph will contain a cycle and they wont form a $t d$-set. So that the only $t d$-sets of two elements is $\left\{v, v_{1}\right\},\left\{v_{1}, v_{i}\right\} ; i=1,2, \ldots, n$. Therefore there are $n$ td-sets of 2 elements. The $t d-$ sets of 3 elements must contain $v$ and $v_{1}$, exactly one vertex from $\left\{v_{2}, \ldots, v_{n}\right\}$. So that $t d-$ sets of cardinality 3 is $n-1$. This completes the proof.
The lollipop graph $L_{m, n}$ is a graph obtained by joining one vertex of a complete graph $K_{m}$ to the end vertex of a path $P_{n}$ with an edge. When $m=1, L_{m, n}$ is the path $P_{n+1}$. For $m \geq 2$ we have the following result.

Theorem 2.17. The tree domination polynomial of the lollipop graph $L_{m, n}$ for $m \geq 2$ is $x^{n}+m x^{n+1}+(m-1) x^{n+2}$.

Proof. $\gamma_{t d}\left(L_{m, n}\right)=n$. Let $\left\{v_{1}, v_{2}, \ldots v_{m}\right\}$ be the vertices of the complete graph $K_{m}$ and $\left\{u_{1}, u_{2}, \ldots u_{n}\right\}$ be the vertices of the path $P_{n}$ and $v_{1} u_{1}$ be the edge joining them. The vertices $u_{1}, \ldots, u_{n-1}$ and $v_{1}$ must be a part of every $t d-$ set, otherwise the induced graph will not be a tree.There is only one $t d-$ set of $n$ elements namely $S=\left\{v_{1}, u_{1}, \ldots u_{n-1}\right\}$. The $t d-$ sets of cardinality $n+1$ are $S \cup\left\{u_{n}\right\}, S \cup\left\{v_{j}\right\} ; j=2, \ldots, m$. Now any three vertices of $K_{m}$ form a cycle; so that no $t d-$ set can contain more than 2 vertices from $\left\{v_{1}, \ldots, v_{m}\right\}$. Thus the only $t d$ - sets of $n+2$ elements are $S \cup\left\{u_{n}, v_{j}\right\}, j=2, \ldots, m$. So we conclude that $D_{t d}\left(L_{m, n}, x\right)=x^{n}+m x^{n+1}+(m-1) x^{n+2}$.This completes the proof.

Note 2.18. Theorem 2.16 is a particular case of the above theorem ; $n=1$.

The bipartite cocktail party graph $B_{n}$ is the graph obtained by removing a perfect matching from the complete bipartite graph $K_{n, n}$. This graph $B_{n}$ is connected iff $n \geq 3$.

Theorem 2.19. $D_{t d}\left(B_{n}, x\right)=\left[\left(\begin{array}{l}n \\ 2\end{array}\right)\left(\begin{array}{l}n \\ 2\end{array}\right)\right] x^{4}+\left[2\left(\begin{array}{l}n \\ 2\end{array}\right)\left(\begin{array}{l}n \\ 3\end{array}\right)\right] x^{5}$, for $n \geq 3$.

Proof. $\gamma_{t d}\left(B_{n}\right)=4$. We have to take 2 vertices from one set of vertices and 2 from other set of vertices otherwise it wont form a $t d$-set. $d_{t d}\left(B_{n}, 4\right)=\left(\begin{array}{l}n \\ 2\end{array}\right)\left(\begin{array}{l}n \\ 2\end{array}\right)$. Also $d_{t d}\left(B_{n}, 5\right)=$ $\left(\begin{array}{l}n \\ 2\end{array}\right)\left(\begin{array}{l}n \\ 3\end{array}\right)+\left(\begin{array}{l}n \\ 2\end{array}\right)\left(\begin{array}{l}n \\ 3\end{array}\right)$. Any set of more than 5 vertices doesnt induce a tree. $\therefore$ It wont form a $t d-$ set.

This completes the proof.

The windmill graph $W_{n}^{(m)}$ is obtained by taking m copies of $K_{n}$ with a vertex in common.

Theorem 2.20. $D_{t d}\left(W_{n}^{(m)}, x\right)=$ $x+m(n-1) x^{2}+\frac{1}{2 !} m(m-1)(n-1)^{2} x^{3}+\frac{1}{3 !} m(m-1)$ $(m-2)(n-1)^{4}+\ldots+(n-1)^{m} x^{m}=x(1+(n-1) x)^{m}$.

Proof. If $v$ is the vertex in common, then $v$ should be a member of every $t d$-set of $W_{n}^{(m)}$ otherwise the induced graph wont be connected. Also we know that the maximum number of elements in a $t d-$ set from a complete graph is 2 . Here $' v^{\prime}$ is common to all $K_{n}$, So that any $t d-$ set can include at most one more vertex other than $v$. As there are $m$ copies of $K_{n}$ and $m(n-1)$ vertices other than $v$, we have $m(n-1)$ choices for choosing one vertex, $\frac{m(n-1)(m-1)(n-1)}{2 !}$ choices for choosing two vertices from two different copies of $K_{n}-v$, $\frac{m(n-1)(m-1)(n-1)(m-2)(n-1)}{3 !}$ choices for choosing three vertices from three different $K_{n}-v$, and so on. Thus for $2 \leq k \leq m$, 
the coefficient of $x^{k}$ is $\frac{m(m-1) \ldots(m-(k-2))(n-1)^{k-1}}{(k-1) !}$. Hence the result.

Dutch windmill graph $D_{n}^{(m)}$ is a windmill graph $W_{n}^{(m)}$ with $\mathrm{n}=3$.

Theorem 2.21. $D_{t d}\left(D_{n}^{(m)}, x\right)=$

$x+\left[m\left(\begin{array}{l}2 \\ 1\end{array}\right)\right] x^{2}+\left[m\left(\begin{array}{l}2 \\ 1\end{array}\right)\right]\left[(m-1)\left(\begin{array}{l}2 \\ 1\end{array}\right)\right] x^{3}$

$+\left[m\left(\begin{array}{l}2 \\ 1\end{array}\right)\right]\left[(m-1)\left(\begin{array}{l}2 \\ 1\end{array}\right)\right]\left[(m-2)\left(\begin{array}{l}2 \\ 1\end{array}\right)\right] x^{4}+\ldots$

$+\left[m\left(\begin{array}{l}2 \\ 1\end{array}\right)\right]\left[(m-1)\left(\begin{array}{l}2 \\ 1\end{array}\right)\right]\left[(m-2)\left(\begin{array}{l}2 \\ 1\end{array}\right)\right] \ldots\left[(m-(m-1))\left(\begin{array}{l}2 \\ 1\end{array}\right)\right] x^{m+}$

Proof. Let $v$ be the common vertex of all $K_{3}$. Now we consider all td-sets of order $i, 1 \leq i \leq m+1$. $\mathbf{i}=\mathbf{1}$ : There is only one td-set of order 1 namely $\{v\}$. $\mathbf{i}=\mathbf{2}$ : One vertex should be $' v^{\prime}$ and the other one can be choosen from any of the $K_{3}$ ie; we have $m\left(\begin{array}{l}2 \\ 1\end{array}\right)$ choices exists in this category. So $\exists m\left(2 C_{1}\right)$ td-sets of order 1 . $\mathbf{i}=3$ : Here we have the freedom to choose 2 vertices from 2 different $K_{3}$. $\therefore$ There are $\left[m\left(\begin{array}{l}2 \\ 1\end{array}\right)\right]\left[(m-1)\left(\begin{array}{l}2 \\ 1\end{array}\right)\right]$ td-sets exists. When $\mathrm{i}=4$ we have $\left[m\left(\begin{array}{l}2 \\ 1\end{array}\right)\right]\left[(m-1)\left(\begin{array}{l}2 \\ 1\end{array}\right)\right]\left[(m-2)\left(\begin{array}{l}2 \\ 1\end{array}\right)\right]$ td-

sets exists. :

Similarly continuing in this way we get, When $i=m+1$ : There are $\left[m\left(\begin{array}{l}2 \\ 1\end{array}\right)\right]\left[(m-1)\left(\begin{array}{l}2 \\ 1\end{array}\right)\right]\left[(m-2)\left(\begin{array}{l}2 \\ 1\end{array}\right)\right] \ldots\left[(m-(m-1))\left(\begin{array}{l}2 \\ 1\end{array}\right)\right]$ td-sets exists.

This completes the proof.

There are certain class of graphs having no $t d-$ set. One such class is that of armed crown graphs. An armed crown $C_{n} \odot P_{m} \ldots \ldots \ldots \ldots . . . . . . F o r ~ n>2, C_{n} \odot P_{m}$ cannot have a $t d-$ set because, all vertices in $C_{n}$ are to be included for connection, but then the induced graph can never be a tree. For any graph $G$ and for any $n \geq 3$, the corona graphs $K_{n} \circ G$ and $C_{n} \circ G$ and in general $H \circ G$, for a graph $H$ containing a cycle, all belong to this category. In fact it is clear that the corona $H \circ G$ have a $t d$ - set if and only if $H$ is a tree.

The $t d$ - polynomial of the corona of a tree $G$ with a complete graph $K_{m}$ can be find out by using the arguments used to prove theorem 2.20. Then 2.20 deals with the windmill graph $\left.W_{n}^{(} m\right)$ which is $m$ copies of $K_{n}$ with a common vertex, the common vertex is in every $t d-$ set. Here all the vertices of $G$ are in every $t d-$ set, and at each vertex we have a copy of $K_{n+1}$.

Theorem 2.22. For any tree $G$ with $n$ vertices, $D_{t d}\left(G \circ K_{1}, x\right)=$ $\left(x^{2}+x\right)^{n}$

Proof. Let $H=t_{n} \circ K_{1}$. Then obviously $\gamma_{t d}(H)=n$ and it is clear that there are $\left(\begin{array}{l}n \\ i\end{array}\right)$ possibilities to extend the tree dominating set into td-sets of size $n+1 . \therefore D_{t d}(H, x)=x^{n}+$ $\left(\begin{array}{c}n \\ 1\end{array}\right) x^{n+1}+\left(\begin{array}{l}n \\ 2\end{array}\right) x^{n+2}+\ldots+x^{2 n}=x^{n}\left[1+\left(\begin{array}{l}n \\ 1\end{array}\right) x+\left(\begin{array}{l}n \\ 2\end{array}\right) x^{2}+\right.$ $\ldots+x^{n}=x^{n}\left[\sum_{k=0}^{n}\left(\begin{array}{l}n \\ k\end{array}\right) x^{k}\right]=\left(x^{2}+x\right)^{n}$.

This completes the proof.
Theorem 2.23. If $G$ is any tree with $n$ vertices then $D_{t d}(G \circ$ $\left.K_{m}, x\right)=x^{n}+\left(\begin{array}{c}n m \\ 1\end{array}\right) x^{n+1}+\left[\left(\begin{array}{c}n m \\ 1\end{array}\right)\left(\begin{array}{c}(n m-(m+1)) \\ 1\end{array}\right)\right] x^{n+2}$

$+\left[\left(\begin{array}{c}n m \\ 1\end{array}\right)\left(\begin{array}{c}(n m-(m+1)) \\ 1\end{array}\right)\left(\begin{array}{c}(n m-2(m+1)) \\ 1\end{array}\right)\right] x^{n+3}+\ldots$

$+\left[\left(\begin{array}{c}n m \\ 1\end{array}\right)\left(\begin{array}{c}(n m-(m+1)) \\ 1\end{array}\right)\left(\begin{array}{c}(n m-2(m+1)) \\ 1\end{array}\right)+\ldots+\left(\begin{array}{c}(n m-(m-1)(n+1)) \\ 1\end{array}\right)\right] x^{2 n}$.

Theorem 2.24. For a tree $G$ with $n$ vertices $D_{t d}\left(G \circ \overline{K_{m}}, x\right)=$ $x^{n}(1+x)^{m n}$.

Proof. If $\overline{K_{m}}$ denote the complement of $K_{m}$, then the graph $G \circ \overline{K_{m}}$ is a graph with a star $K_{1, m}$ at each vertex $v$ in $G$; with $v$ as center. If $G$ is a tree on $n$ vertices, then $G \circ \overline{K_{m}}$ on $n+n m$ vertices. The only $\gamma_{t d}$ set is the vertex set of $G$ and all its supersets will become a $t d$ - set. Therefore there are $\left(\begin{array}{c}n m \\ i\end{array}\right)$ $t d-$ sets of cardinality $n+i$. Thus we have proved the theorem. For a tree $G$ with $n$ vertices $D_{t d}\left(G \circ \overline{K_{m}}, x\right)=x^{n}(1+x)^{m n}$.

This completes the proof.

The joint sum of two cycles $C_{m}$ and $C_{n}$ is a new graph obtained by joining a vertex of $C_{n}$ to a vertex of $C_{m}$ with new edge.

Theorem 2.25. Let $G$ be the joint sum of two cycles $C_{m}$ and $C_{n}$.

Then $\gamma_{t d}(G)=m+n-4$ and the number of minimum tree dominating set of $G$ is $m n-2(m+n)+4$.

Proof. Assume that $G$ be the joint sum of two cycles $C_{m}$ and $C_{n}$. It is clear that the two joint vertices from $C_{m}$ to $C_{n}$ must include in any minimum tree dominating set of $G$. Therefore the minimum tree dominating set of $G$ is $m+n-4$. This follows that $\gamma_{t d}(G)=m+n-4$.

Let $u$ and $v$ be the joint vertices of $C_{m}$ and $C_{n}$ respectively. Let $N(u)=\left\{u_{1}, u_{2}, v\right\}$ and $N(v)=\left\{v_{1}, v_{2}, u\right\}$. To select $m-2$ vertices from $C_{m}$ and $n-2$ vertices from $C_{n}$ to be minimum tree dominating set of $G, u$ and $v$ must belong to any minimum tree dominating set of $G$. So from $C_{m}$ number of ways selected vertices is equal to the number of edges in $C_{m}$ excluding the edges $u u_{1}$ and $u u_{2}$. So there are $m-2$ choices to select vertices from $C_{m}$. Similarly there are $n-2$ choices to select vertices from $C_{n}$. Hence the number of minimum $t d$-sets in $G$ is $m n-2(m+n)+4$.

This completes the proof.

The following results are easy to prove.

Theorem 2.26. Let $G$ be the joint sum of two cycles $C_{m}$ and $C_{n}$. Then

$$
\begin{aligned}
& \text { 1. } d_{t d}(G, m+n-3)=(m-2)(n-1)+(m-1)(n-2) . \\
& \text { 2. } d_{t d}(G, m+n-2)=(m-1)(n-1) .
\end{aligned}
$$

Theorem 2.27. Let $G$ be the joint sum of two cycles $C_{m}$ and $C_{n}$. Then

displaystyle $D_{t d}(G, x)=(m-2)(n-2) x^{m+n-4}+[(m-2)(n-$ 1) $+(m-1)(n-2)] x^{m+n-3}+(m-1)(n-1) x^{m+n-2}$.

Proof. The proof is very clear by Theorems 23 and 24 . 
Theorem 2.28. Let $G$ be the joint sum of two copies of $C_{n}$. Then

$D_{t d}(G, x)=(n-2)^{2} x^{2 n-4}+2(n-2)(n-1) x^{2 n-3}+(n-1)^{2} x^{2 n-2}$.

If we evaluate the graph polynomial at a particular point can give some interesting information about the graph. Also there are many studies doing in this area.

It is clear that $D_{t d}(G, 1)$ is the number of tree dominating sets of $G$, and $D_{t d}(G,-1)$ is the difference between the number of tree dominating sets of even size and the number of tree dominating sets of odd size. In the results given below we got the value of td-polynomial of some standard graphs at -1 .

Proposition 2.29. For any path $P_{n}, n \geq 3$ vertices, $D_{t d}\left(P_{n},-1\right)=$ 0 .

Proof. The proof is clear from Theorem 2.3.

Proposition 2.30. For a cycle $C_{n}, D_{t d}\left(C_{n},-1\right)=0$.

Proof. The proof is followed by Theorem 2.4. Also by using the Theorem 4 we get the following result.

Proposition 2.31. For any star graph $K_{1, t}, t \geq 2$ with $t+1$ vertices, $D_{t d}\left(K_{1, t},-1\right)=0$.

Similarly by Theorem 2.5 , we get the following result.

Proposition 2.32. For a complete graph $K_{n}, D_{t d}\left(K_{n},-1\right)=$ $\frac{n(n-3)}{2}$

Proposition 2.33. 1. For any tree $t_{n}, D_{t d}\left(t_{n} \circ K_{1},-1\right)=$ 0.

2. For any tree $t_{n}, D_{t d}\left(t_{n} \circ \overline{K_{m}},-1\right)=0$.

\section{Conclusion}

We have studied the $t d$ - polynomial of many important classes of graphs, and continue for other classes of graphs, and derived graphs, using graphs operations like join, corona,etc. In this area our main task is to relate the $t d-$ polynomial of the derived graph with that of its underlying graphs. Also we are trying to find whether we can extract the important features of a graph from the parameters related to its $t d-$ polynomial.

\section{References}

[1] J. A. Bondy, U.S.R. Murty; Graph Theory; Springer, 2008.

[2] M.Shikhi, V.Anilkumar; Common Neighbour Polynomial Of Graphs; Far East Journal Of Mathematical Sciences; Pushpa Publishing House, 102(6)(2017), 1201-1221.

[3] P. Raji, K. Preethi; On Mixed Tree Domination Number; Proceedings of the International Conference on Number Theory, P.D.E. and Geometry; Department of Mathematics, University of Calicut(2009), 249-255.
[4] P. Raji, K. Preethi; On Mixed Tree Domination Number; Advances and Applications in Discrete Mathematics;Pushpa Publishers, 9(1)(2012), 45-55.

[5] Dhananjayamurthy B.V.;PhD Thesis Some Contributions to the Theory of Domination In Graphs., 2015.

[6] Saeid Alikhani and Yee-hock Peng,Introduction to domination polinomial of a graph, arXiv: 0905. $2251 \mathrm{~V} 1$ (2009).

[7] Preethi Kuttipulackal and Rafia Yoosuf;The mixed Tree Domination Polynomial,Contemporary Studies In Discrete Mathematics; CSDM Publishers, Vol.2, No.3(2018), 15-20.
Malaya Journal of Matematik

ISSN(O):2321 - 5666

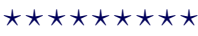

